Proceedings

\title{
Information Geometry Conflicts With Independence ${ }^{\dagger}$
}

\author{
John Skilling $(\mathbb{B}$
}

Maximum Entropy Data Consultants Ltd., Kenmare, Ireland; skilling@eircom.net

+ Presented at the 39th International Workshop on Bayesian Inference and Maximum Entropy Methods in Science and Engineering, Garching, Germany, 30 June-5 July 2019.

Published: 5 December 2019

Abstract: Information Geometry conflicts with the independence that is required for science and for rational inference generally.

Keywords: Information Geometry; metric space; probability distribution

PACS: $02.50 \mathrm{Cw} ; 02.70 \mathrm{Rr}$

\section{Introduction}

Information Geometry [1] assigns a geometrical relationship between probability distributions, using the local curvature (Hessian) of the Kullback-Leibler formula

$$
H(\mathbf{p} ; \mathbf{q})=\sum_{i} p(i) \log \frac{p(i)}{q(i)}
$$

as the covariant geometrical metric tensor [2,3] between $\mathbf{q}$ and $\mathbf{p}$. On a $n$-dimensional manifold $\mathbf{p}(\theta)$ specified by parameters $\theta^{1}, \ldots, \theta^{n}$, this $n \times n$ Riemannian metric $g$ is

$$
g_{j k}(\theta)=\sum_{i} p(i \mid \theta) \frac{\partial \log p(i \mid \theta)}{\partial \theta^{j}} \frac{\partial \log p(i \mid \theta)}{\partial \theta^{k}} \quad\left(\text { or } \int d t p(t \mid \theta) \ldots\right. \text { in continuum form) }
$$

Geodesic lengths $\ell$ and invariant volumes $V$ follow from $(d \ell)^{2}=\sum g_{j k} d \theta^{j} d \theta^{k}$ and $d V=\sqrt{\operatorname{det} g} d^{n} \theta$.

Necessarily, lengths are symmetric $\ell(\mathbf{p}, \mathbf{q})=\ell(\mathbf{q}, \mathbf{p})$ between source and destination, so cannot be isomorphic to $H$ which is from-to asymmetric. Yet (1) is the only connection which preserves independence of separate distributions, $H(\mathbf{x} \times \mathbf{p} ; \mathbf{y} \times \mathbf{q})=H(\mathbf{x} ; \mathbf{y})+H(\mathbf{p} ; \mathbf{q})$. Specifically, when $H$ is used to assign an optimal $\mathbf{p}$ (meaning minimally distorted from $\mathbf{q}$ ) under constraints, that "maximum entropy" selection also depends on separate optimisation $\mathbf{x}$-from-y unless $H$ has the form (1) [4,5].

It follows that any imposed geometrical connection must introduce interference between supposedly separate distributions. That behaviour is incompatible with the practice of scientific inference, and is confirmed by a counter-example. 


\section{Counter-Example}

Consider the 2-parameter family of probability distributions [6]

$$
\mathbf{p}_{v w}(t(\bmod 1))= \begin{cases}f\left(\frac{t-v}{w}\right) & \text { for } v<t<v+w \\ f\left(\frac{1+v-t}{1-w}\right) & \text { for } v+w<t<v+1\end{cases}
$$

Parameters $v$ (location) and $w$ (width) lie between 0 and 1 . The function $f$ (Figure 1 ) is monotonically increasing so that it rises from $f(0)$ at $t=v$ to $f(1)$ at $t=v+w(\bmod 1)$ before falling back to $f(0)$ at $t=v+1(\bmod 1)$. It is positive and normalised to $\int_{0}^{1} f(u) d u=1$ so that the $p_{v w}(\cdot)^{\prime}$ s can be probability distributions on the interval $(0,1)$ - which could model growth and decay in a periodic system.

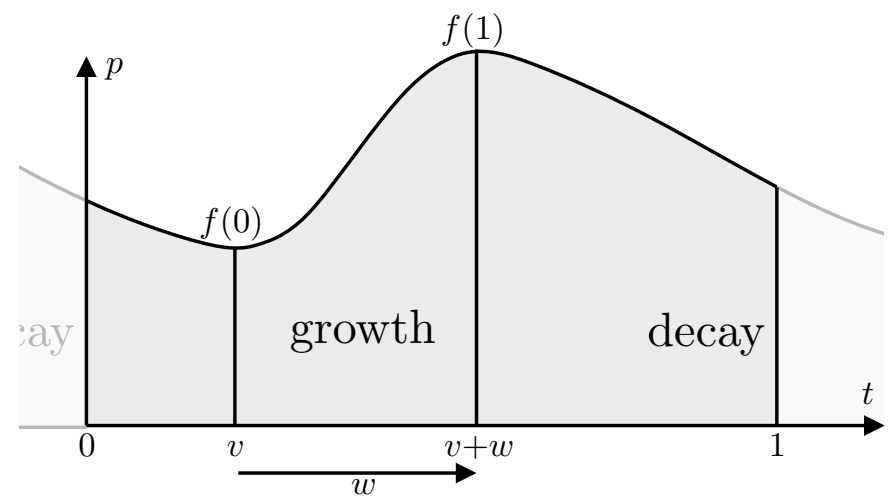

Figure 1. Does $v$ affect $w$ ?

\subsection{Two Parameters $v$ and $w$}

The $2 \times 2$ information-geometry metric evaluates to

$$
\left[\begin{array}{ll}
g_{v v} & g_{v w} \\
g_{w v} & g_{w w}
\end{array}\right]=\frac{1}{w(1-w)} \int_{0}^{1}\left[\begin{array}{cc}
1 & u \\
u & u^{2}
\end{array}\right] \frac{f^{\prime}(u)^{2}}{f(u)} d u=\left[\begin{array}{cc}
A & B \\
B & C
\end{array}\right] / w(1-w)
$$

where $A, B, C$ are constants. The table shows their values for two example functions. The first is easy to

\begin{tabular}{|c|c|c|}
\hline$f(u)$ & $e^{u} /(e-1)$ & $\left(8+6 u^{2}-4 u^{3}\right) / 9$ \\
\hline$A$ & $e-1=1.71828$ & $\frac{11}{6} \log 2+\frac{5}{6} \log 5-\sqrt{15}\left(\arctan \frac{5}{\sqrt{ } 15}-\arctan \frac{1}{\sqrt{ } 15}\right)$ \\
\hline B & $1=1.00000$ & $\frac{89}{12} \log 2-\frac{25}{12} \log 5-\frac{\sqrt{15}}{6}\left(\arctan \frac{5}{\sqrt{ } 15}-\arctan \frac{1}{\sqrt{ } 15}\right)-\frac{4}{3}=0.02909$ \\
\hline C & $e-2=0.71828$ & $\frac{251}{24} \log 2+\frac{5}{24} \log 5+\frac{13 \sqrt{15}}{12}\left(\arctan \frac{5}{\sqrt{ } 15}-\arctan \frac{1}{\sqrt{ } 15}\right)-\frac{31}{3}=0.01636$ \\
\hline
\end{tabular}
integrate while the second has vanishing slope $f^{\prime}(0)=f^{\prime}(1)=0$ at the joins (as in Figure 1).

The invariant volume element follows as

$$
d V=\sqrt{\operatorname{det} g} d v d w=\frac{\sqrt{A C-B^{2}}}{w(1-w)} d v d w
$$


where, by construction, $A C-B^{2}>0$. The total invariant volume is infinite.

$$
V=\int_{0}^{1} d v \int_{0}^{1} d w \sqrt{\operatorname{det} g}=\infty
$$

\subsection{One Parameter $w$}

If $v$ had been fixed, $\mathbf{p}$ would have been confined to a submanifold $\mathbf{p}_{w}(\cdot)$ parameterised by $w$ alone. The information-geometry metric reduces to

$$
g_{w w}=\frac{1}{w(1-w)} \int_{0}^{1} u^{2} \frac{f^{\prime}(u)^{2}}{f(u)} d u=\frac{C}{w(1-w)}
$$

The invariant volume element follows as

$$
d V=\sqrt{g_{w w}} d w=\left(\frac{C}{w(1-w)}\right)^{1 / 2} d w
$$

where, by construction, $C>0$. The total invariant volume is finite.

$$
V=\int_{0}^{1} \sqrt{g_{w w}} d w=\pi C^{1 / 2}
$$

\subsection{Comparison of One and Two Parameters}

Both shape ((5) versus (8)) and integral ((6) versus (9)) over $w$ differ qualitatively according to whether or not $v$ is held fixed.

Treatment of $v$ influences invariant volumes over $w$

[Geometry]

That is a mathematical fact of information geometry.

\subsection{Science}

For scientific application, (3) defines a wraparound translation-invariant model in which $v$ does not affect $w$.

Treatment of $v$ should not influence inference about $w$

[Science]

That is a science requirement. Any observational consequence of information-geometry's invariant volumes would be rejected by the informed scientist. If there were such consequence, then observation of width $w$ could be used to infer something about location $v$, contrary to the intention of the formulation.

\section{Conclusions}

Information geometry is not science. It denies the independence of separate parameters even though such independence is a fundamental requirement of scientific inquiry. The assumption of a geometrical connection between distributions is unnecessary for science and it fails under test.

Information geometry is a self-consistent mathematical structure which (like any other piece of mathematics) may find specialised application within science, but is not fundamental to it. The only fundamental connection is the Kullback-Leibler, which is from-to asymmetric hence not geometric.

Funding: This research received no external funding.

Acknowledgments: This investigation has been refined by many conversations, in particular with Ariel Caticha.

Conflicts of Interest: The author declares no conflict of interest. 


\section{References}

1. Amari, S. Differential-geometrical methods in statistics. In Lecture Notes in Statistics; Springer-Verlag: Berlin, Germany, 1985.

2. Fisher, R. A. Theory of statistical estimation Proc. Camb. Philos. Soc. 1925, 122, 700-725.

3. Rao, C.R. Information and the accuracy attainable in the estimation of statistical parameters. Bull. Calcutta Math. Soc. 1945, 37, 81-89.

4. Shannon, C.F. A Mathematical theory of Communication. Bell Syst. Tech. J. 1948, 27, 379-423, $623-656$.

5. Knuth, K.H.; Skilling, J. Foundations of Inference. Axioms 2012, 1, 38-73.

6. Skilling, J. Critique of Information Geometry. AIP Conf. Proc. 2013, 1636, 24-29.

(C) 2019 by the authors. Licensee MDPI, Basel, Switzerland. This article is an open access article distributed under the terms and conditions of the Creative Commons Attribution (CC BY) license (http:/ / creativecommons.org/licenses/by/4.0/). 